\title{
Mass Spectrometry in Proteomics: Technologies, Methods, and Research Applications for the Life Sciences
}

\author{
Paolo Nanni§ ${ }^{\S}$ Peter Gehrig ${ }^{\S}$, and Ralph Schlapbach*
}

\begin{abstract}
Mass spectrometry is a powerful tool in the hand of life science researchers, who constantly develop and apply new methods for the investigation of biomolecules, such as proteins, peptides, metabolites, lipids, and glycans. In this review, we will discuss the importance of mass spectrometry for the life science sector, with a special focus on the most relevant current applications in the field of proteomics. Moreover, we will comment on the factors that research groups should consider when setting up a mass spectrometry laboratory, and on the fundamental role played by academic core facilities and industrial service providers.
\end{abstract}

Keywords: Core facilities · Life sciences · Mass spectrometry · Protein analysis · Proteomics

\section{Mass Spectrometry in the Life Sciences}

Mass spectrometry (MS) has evolved from a technology to characterize small chemical molecules to an indispensable tool in the hands of researchers in all life sciences. Thanks to their analytical power and versatility, MS-based methods enable the investigation of proteins, peptides, metabolites, lipids, glycans, and biomolecules in general. Many life science studies involve MS at some step, either to check the quality of molecules (e.g. identity of a protein), to characterize them in detail (e.g. glycan profiling of an antibody), to discover new markers for biological processes (e.g. metabolites involved in a specific disease), or to validate findings obtained by other analytical means (e.g. increased level of specific protein post-translational modifications). Mass spectrometry is increasingly relevant also in the biopharmaceutical sector, where the filing of new biotechnological products follows detailed characterization procedures defined in the ICH guidelines (International Council for Harmonisation of Technical Requirements for Pharmaceuticals for Human Use).

Given its broad range of applications, MS is a field of research and technology development on its own, with branches concentrating on different life science disciplines, and more specifically on distinct types of biomolecules and applications. Examples of these disciplines are proteomics, metabolomics, lipidomics and glycomics, which focus on the large-scale studies of proteins, metabolites, lipids and glycans, respectively. The analysis of these classes of biomolecules requires different analytical workflows and instrument capabilities, but also distinct technical and scientific expertise.

While some specialized research groups have their own mass spectrometers for the development and improvement of MSmethods, most life science research groups use mass spectrometry primarily to answer a specific biological question, for which they rely on the infrastructure and expertise of collaborators, academic core facilities, or commercial service providers.

In the following, we will describe the most important technological and analytical developments that shaped the field of life science mass spectrometry, with a particular focus on the field of proteomics. We will provide examples on how

${ }^{\star}$ Correspondence: Prof. Dr. R. Schlapbach, E-mail: ralph.schlapbach@fgcz.uzh.ch Functional Genomics Center Zurich, University of Zurich/ETH Zurich;

§Equal contribution proteomics is applied to unravel cellular mechanisms, to analyze protein interaction networks, or to characterize protein structures. We will also comment on the importance of data analysis and bioinformatics.

Finally, we will discuss the challenges associated to the provision of life science MS services and support, and the relevance of training life scientists in mass spectrometry.

\section{Technological Developments Enabling today's Use of Mass Spectrometry}

Already in 1959, the first application of electron ionization mass spectrometry to peptide sequencing was published by the group of K. Biemann. ${ }^{[1]}$ Nearly two decades later, this technique was used to sequence entire proteins exclusively by mass spectrometry, or in combination with DNA sequencing data. ${ }^{[2]}$ Mass spectrometry was limited to small and thermostable molecules for a long time because of the lack of soft desorption and ionization methods. Biological mass spectrometry was revolutionized by the development of ionization methods that allowed the ionization of large, involatile, and fragile molecules.

The almost simultaneous introduction of electrospray ionization (ESI ${ }^{[3]}$ and matrix-assisted laser desorption (MALDI) ${ }^{[4]}$ for the analysis of peptides and intact proteins represented a major advance and brought mass spectrometry of these large biomolecules closer to real applications. Online coupling of liquid chromatography (LC) to an ESI-MS instrument enabled sequencing of femtomole amounts of peptides by D. Hunt $e t$ al. already in 1992..$^{[5]}$ Shortly afterwards, J. Yates et al. introduced a software tool (SEQUEST) to correlate tandem mass spectra of peptides with amino acid sequences in protein databases. ${ }^{[6]}$ This combination of LC, ESI mass spectrometry, and protein sequence database searches remains at the heart of mass spectrometrybased peptide and protein analysis.

Mass spectrometry technology has been greatly improved in terms of sensitivity, acquisition speed, mass accuracy and resolution over the past 30 years. A variety of different mass analyzers, hybrid instruments containing more than one analyzer, and MS/ MS fragmentation methods have been developed. Electrospray ion sources were first connected to single and triple quadrupole mass spectrometers. In a tandem mass spectrometry experiment using a triple quadrupole instrument, the first quadrupole is used as a mass filter to select precursor ions with specific mass-tocharge $(\mathrm{m} / \mathrm{z})$ values, the intermediate quadrupole is used as a 
collision cell, and the third one is utilized to perform analyses of fragment ions.

Quadrupole ion traps and later linear ion traps equipped with electrospray ion sources are another type of mass spectrometers that have been and still are in wide use for peptide and protein analysis. ${ }^{[7]}$ Their mass resolution and accuracy are relatively low, but they provide particularly rapid and highly sensitive fragment ion scanning. MS instruments with high resolution and high mass accuracy analyzers were developed, most notably quadrupole time-of-flight ${ }^{[8]}$ and Orbitrap ${ }^{[9]}$ mass spectrometers, which also provided high sensitivity and high acquisition speed. These substantial improvements increased confidence in the identification of peptides and post-translational modifications.

Different types of hybrid mass spectrometers containing more than one ion analyzer or mass filter have been created. Among them, quadrupole Orbitrap mass spectrometers have become particularly popular in proteomics research. ${ }^{[10]}$ These instruments combine precursor ion selection in a quadrupole mass filter and ion fragmentation in a collision cell with highly accurate mass detection of ions in an Orbitrap mass analyzer. A related and even more complex instrument is the quadrupole/linear ion trap/ Orbitrap tribrid mass spectrometer. ${ }^{[11]}$ In a standard proteome analysis performed on this instrument, intact peptide ions are analyzed in the Orbitrap with high mass accuracy, and the less abundant fragment ions are typically analyzed in the linear ion trap, which provides low detection limits even at high scan speed.

In recent years, there has been growing interest in the combination of MS and ion mobility spectrometry, an analytical technique used to separate ions in the gas phase based on their mobility in a carrier buffer gas. A trapped ion mobility spectrometry (TIMS) device was coupled to a quadrupole time-of-flight mass spectrometer, to provide an added dimension of separation in addition to LC and MS: precursor ions can be accumulated in parallel and sequentially released from the TIMS device based on their ion mobility. A new method called parallel accumulation serial fragmention (PASEF) was developed, which provides higher sensitivity and increased numbers of peptide and protein identifications. ${ }^{[12]}$ A different type of ion mobility technique denoted as high field asymmetric waveform ion mobility spectrometry (FAIMS) has been implemented to separate peptides or other analytes between liquid chromatography and mass spectrometry. ${ }^{[13]}$ As a means of online fractionation, it enhances detection of analytes in complex samples by selectively passing through different sets of ions upon changes of the electric field.

Collision-induced dissociation (CID), where neutral gas atoms or molecules collide with analyte ions, is one of the most commonly used fragmentation methods. CID fragmentation mainly results in cleavage of the peptide backbone amide bonds. Electron transfer dissociation (ETD) fragmentation ${ }^{[14]}$ and related fragmentation methods are particularly suitable for the analysis of labile protein modifications. ETD, which involves the transfer of an electron to multiply charged peptide ions, causes peptide backbone cleavage, while leaving labile posttranslational modifications largely intact. High-energy collisional dissociation (HCD), ${ }^{[15]}$ which is conceptually related to other beam-type implementations of CID in collision cells, became available in Orbitrap mass spectrometers. Furthermore, a combination of HCD and ETD, electron-transfer and higherenergy collisional dissociation known as $\mathrm{EThcD}^{[16]}$ was implemented in hybrid ion trap/Orbitrap mass spectrometers. EThcD spectra show fragment ions resulting from both HCD and ETD fragmentation processes.

\section{Mass Spectrometry-based Proteomics}

ESI and MALDI mass spectrometry were and still are useful in determining the molecular weights of biomolecules, and in the characterization of single, purified proteins. A number of developments in analytical technologies and bioinformatics allowed the application of MS-based protein analyses to more complex samples and eventually enabled actual proteomic studies. As the name implies, proteomics (from protein and genomics) aims to investigate the entire content of proteins present in cells, tissues, body fluids, whole organisms, or in any type of biological sample. Mass spectrometry-based proteomics is now the most comprehensive approach for quantitative profiling of proteins, their interactions and localizations in cells, and for the identification of protein modifications. Proteomics has benefitted greatly from the genetic information of various genome projects and from the availability of commercial instruments enabling automated selection and fragmentation of precursor ions during LC-MS analyses.

The basic proteomics workflow includes the following steps: protein extraction from biological samples, cleavage with trypsin or with other proteolytic enzymes, and online LC-MS analysis of the resulting peptides. For typical data-dependent acquisition (DDA) measurements, also called 'shotgun proteomics', a survey scan is performed, and the $\mathrm{N}$ precursor ions of highest abundance are then selected for MS/MS analysis based on their $\mathrm{m} / \mathrm{z}$ values. Eventually, the sequences of peptides and associated proteins are identified by database search algorithms (Fig. 1).
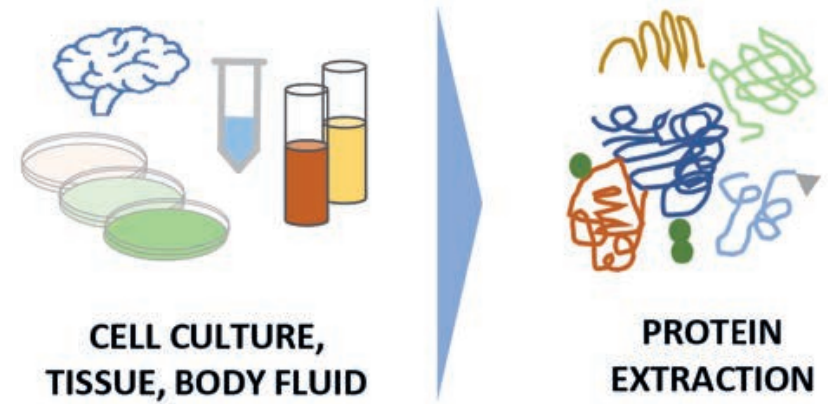

\section{PROTEIN EXTRACTION}
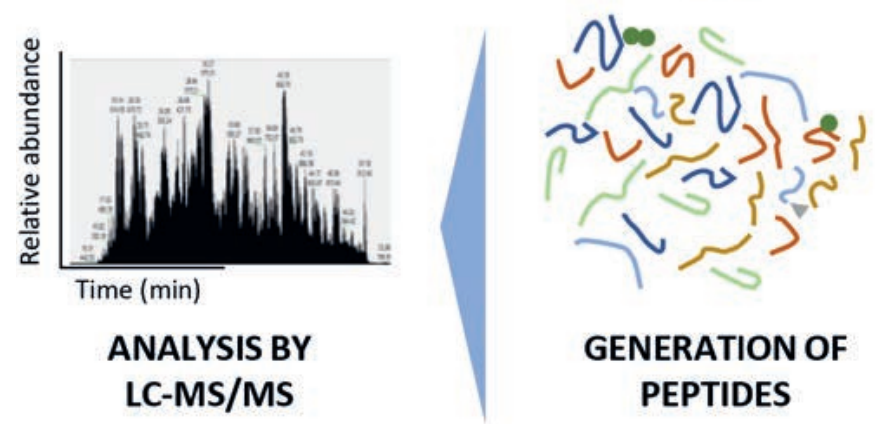

GENERATION OF PEPTIDES

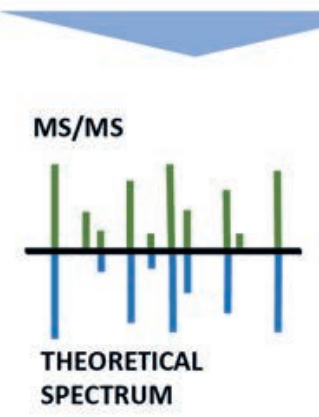

SEARCH

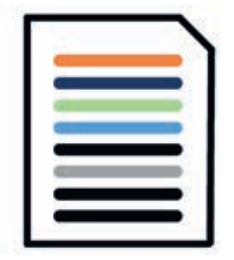

PROTEIN IDENTIFICATION

Fig. 1. Overview of a typical proteomics workflow. 
Prefractionation of proteolytic peptides, for example by using alternative chromatographic methods is a possibility to achieve even deeper proteome coverage. ${ }^{[17]}$ Furthermore, comprehensive analysis of protein modifications typically requires a specific enrichment of modified peptides.

\section{Major Proteomics Applications and Methods}

In this section, we will describe the most common proteomics workflows, together with some examples of their application to research questions (summarized in Table 1).

Table 1. Examples of proteomics methods and their application to research questions

\begin{tabular}{|c|c|}
\hline Examples of research questions & Methods \\
\hline $\begin{array}{l}\text { - Identification of protein } \\
\text { content and of differentially } \\
\text { expressed proteins } \\
\text { - Biomarker discovery } \\
\text { - Study of cellular mechanisms } \\
\text { - Quantification of target } \\
\text { proteins }\end{array}$ & $\begin{array}{l}\text { Protein identification and } \\
\text { quantification: } \\
\text { Metabolic labeling, }{ }^{[18]} \\
\text { chemical labeling, }{ }^{[19,20]} \text { label- } \\
\text { free analysis }{ }^{[21]} \text { targeted } \\
\text { quantification }\left(\mathrm{SRM},{ }^{[22]} \text { PRM, }{ }^{[23]}\right. \\
\left.\text { DIA }^{[24]}\right)\end{array}$ \\
\hline $\begin{array}{l}\text { - Analysis of protein complexes } \\
\text { and interaction networks }\end{array}$ & $\begin{array}{l}\text { Interaction proteomics: } \\
\text { Affinity purification (AP-MS); }{ }^{[25]} \\
\text { proximity labeling (BioID, },^{[26]} \\
\text { APEX }{ }^{[27]} \text { ) }\end{array}$ \\
\hline $\begin{array}{l}\text { Understanding proteins } \\
\text { - Signaling } \\
\text { - Turnover } \\
\text { - Interaction } \\
\text { - Crosstalk } \\
\text { - Localization } \\
\text { - Enzymatic activity }\end{array}$ & $\begin{array}{l}\text { Post-translational } \\
\text { modifications (PTMs): } \\
\text { Phosphorylation, }{ }^{[28]} \\
\text { acetylation, }{ }^{[29]} \text { methylation, }{ }^{[30]} \\
\text { ubiquitination, }{ }^{[31]} \text { ADP- } \\
\text { ribosylation, }{ }^{[32-34]} \\
\text { glycosylation }{ }^{[35,36]}\end{array}$ \\
\hline $\begin{array}{l}\text { Characterization of } \\
\text { protein structures }(e . g . \\
\text { biopharmaceuticals })\end{array}$ & $\begin{array}{l}\text { Protein characterization: } \\
\text { Disulfide bridges, }{ }^{[37]} \mathrm{N} \text {-Terminal } \\
\text { analysis }^{[38]}\end{array}$ \\
\hline $\begin{array}{l}\text { - Protein conformation } \\
\text { - Protein stability } \\
\text { - Drug-target deconvolution } \\
\text { - Stoichiometry and structure of } \\
\text { protein complexes }\end{array}$ & $\begin{array}{l}\text { Structural proteomics: } \\
\text { Limited proteolysis (LiP); }{ }^{[39]} \\
\text { cross-linking, }{ }^{[40,41]} \text { native } \mathrm{MS}^{[42,43]}\end{array}$ \\
\hline
\end{tabular}

\subsection{Protein Quantification}

\subsubsection{Discovery-based Proteome Quantification}

To understand the functions of proteins, it may be of interest to measure changes of protein abundance upon treatment or other changes of the biological system (e.g. for the discovery of protein biomarkers in clinical samples, or the identification of molecular pathways affected by a drug treatment). A number of quantitative protein profiling approaches have been developed based on the original workflow for protein identification.

Stable isotope labeling using amino acids in cell culture (SILAC) is an accurate and well-established method used to identify and quantify relative changes of proteins in complex samples. ${ }^{[18]}$ SILAC involves in vivo incorporation of heavy ${ }^{13} \mathrm{C}$ or ${ }^{15} \mathrm{~N}$-labeled amino acids into proteins in cell cultures. Cells grown under differing conditions in light or heavy media are then mixed, and all following sample processing and analysis steps are conducted on the combined sample. For this reason, variability due to sample processing is greatly reduced, resulting in particularly accurate protein quantification.

Chemical labeling with isobaric reagents, for example iTRAQ ${ }^{[19]}$ and TMT, ${ }^{20]}$ is a generally applicable alternative to
SILAC. It can be used to quantify relative changes in complex protein samples in a single analysis across multiple experimental conditions, it is applicable with all kinds of samples, and it is not restricted to cell cultures. iTRAQ and TMT reagents are isobaric tags, which consist of a reporter group, a linker and an aminereactive group, which reacts with $\mathrm{N}$-termini of peptides and lysine residues. Unique reporter ions are generated from each tag during MS/MS fragmentation, and the peptide and protein quantification is based on relative reporter ion intensities.

Label-free peptide and protein quantification involves comparing the abundances of peptide ion signals in different samples without the use of isotopic labels. ${ }^{[21]}$ Samples are separately processed and analyzed by LC-MS in label-free quantification experiments. The approach is applicable to biomarker studies in complex proteome samples, but also to single proteins or protein complexes of interest. The label-free quantification experiments have the advantages that the number of samples is not limited, samples can be of any origin, and all MS/MS fragmentation techniques are applicable.

\subsubsection{Targeted Proteome Quantification}

Data-dependent acquisition methods can certainly identify large numbers of peptides and proteins, but complete coverage of specific biological pathways or of functional classes of proteins (for example kinases or transcription factors) remains challenging. The aim of a targeted proteomics experiment is to monitor selected peptides and proteins of interest with even higher sensitivity, reproducibility and quantitative accuracy across multiple samples. In the following paragraph, we describe three widely applied targeted mass spectrometry techniques.

Selected reaction monitoring (SRM) conducted on a triple quadrupole mass spectrometer enables sensitive detection and reproducible quantification of preselected peptides or other analytes. ${ }^{[22]}$ In these experiments, a precursor ion is isolated in the first quadrupole, fragmented in the collision cell, and the previously determined fragment ions are then selected in the third quadrupole and transferred to the detector. In parallel reaction monitoring (PRM) experiments, ${ }^{[23]}$ the third quadrupole of a triple quadrupole is substituted with a high-resolution mass analyzer to permit the parallel detection of all target product ions in one mass analysis. Quadrupole Orbitrap or quadrupole time-of-flight instruments, which provide good precursor ion mass selection and high resolution and mass accuracy, are typically utilized for PRM. Data-independent acquisition (DIA) aims to combine advantages of targeted and untargeted acquisition methods. ${ }^{[24]}$ It involves acquisition of tandem mass spectra by sequentially isolating and fragmenting broad ranges of $\mathrm{m} / \mathrm{z}$, for example 25-Da precursor selection windows. From DIA data sets, peptides and respective proteins are identified and quantified either directly or by targeted data extraction using reference spectra previously obtained by standard data-dependent acquisition.

\subsection{Interaction Proteomics}

\subsubsection{Affinity Purification MS of Protein Complexes}

The analysis of protein complexes and of protein-protein interaction networks is of central importance in biological research. The combination of affinity purification and mass spectrometry (AP-MS) has been applied to characterize numerous protein complexes and even entire protein-protein interaction networks. Comprehensive reviews of affinity purification MS methods and applications are provided in refs [25] and [44]. In summary, proteins of interest (bait proteins) are expressed with an $\mathrm{N}$ - or C-terminal tag, which is then used to purify the bait protein together with interacting proteins. These enriched protein samples are subjected to proteolysis and analyzed. Good controls are required to differentiate interacting proteins from ubiquitous and abundant proteins. The AP-MS technique only generates a list 
of proteins identified in a given sample and does not necessarily reveal the composition of individual protein complexes. Not only protein contaminants, but also weakly interacting binding partners represent a problem in affinity purification and mass spectrometry data. Only protein-protein interactions resistant to lysis and affinity purification conditions can be detected by mass spectrometry.

\subsubsection{Proximity Labeling}

Proximity labeling is an approach to determine proteins in the region around a protein of interest, without requiring high affinity of protein-protein interactions. Two different proximity labeling methods named BioID ${ }^{[26]}$ and $\mathrm{APEX}^{[27]}$ have been developed. Proximity labeling involves enzymatic labeling of proteins or other biomolecules near a protein of interest: by creating a gene fusion between this protein and a labeling enzyme (biotin ligase BirA or ascorbic acid peroxidase) in cells, biotin is attached to proximal proteins. Biotin-marked proteins are then subjected to affinity purification and MS analysis. Proximity labeling has been applied for protein-protein interaction studies and also for identifying components of organelles and cellular structures. Recent developments in the field of proximity dependent biotinylation and applications in proteomics studies are reviewed in ref. [45].

\subsection{Protein Characterization}

Post-translational protein modifications (PTMs) often regulate protein activity or function and eventually modify signaling pathways and cellular processes. Many protein modifications are reversible, and specific enzymes catalyze the addition (for example kinases, acetyltransferases, methyltransferases) or the removal (phosphatases, deacetylases) of modifications. Several hundred types of PTMs have been reported in the literature, ${ }^{[46]}$ but only a few of them have been widely studied, most notably phosphorylation and glycosylation of proteins. Sample preparation methods and workflows for PTM analysis are similar to those for standard peptide and protein analysis, however, comprehensive and proteome-wide detection of PTMs of interest typically requires an enrichment step. Alternatively, single proteins are isolated and characterized with respect to all PTMs. Furthermore, MS/ MS spectra should contain sufficient fragment ion information to identify the modified amino acid residue. Some PTMs with particularly labile structures may require the application of ETD or EThcD fragmentation.

\subsubsection{Protein Phosphorylation}

There are many roles of protein phosphorylation in cell biology. The addition of phosphate groups to serine, threonine, tyrosine, or other amino acids is fast and reversible, and represents an important process for the regulation of protein activity and signal transduction. Mass spectrometry-based phosphoproteomics studies have been essential for our understanding of biological processes altered by protein phosphorylation. ${ }^{[28]}$

A number of enrichment protocols with high specificity for phosphopeptides have been developed, which often result in a phosphopeptide proportion $>90 \%$. The two most commonly utilized methods are immobilized metal ion affinity chromatography (IMAC), for example $\mathrm{Fe}^{3+}$ - and $\mathrm{Ti}^{4+}$-IMAC, and metal oxide affinity chromatography, usually based on titanium dioxide. $\mathrm{Fe}^{3+}$-IMAC-based phosphopeptide enrichment and technical advances in mass spectrometric analysis enabled the first proteome-wide phosphorylation studies published by the research groups of D. Hunt ${ }^{[47]}$ and S. Gygi. ${ }^{[48]}$ Meanwhile, tens of thousands of phosphorylation sites have been identified in large-scale proteomic studies. In principle, both label-free and label-based peptide quantification approaches as well as targeted methods are applicable in phosphoproteomic analyses. However, quantitative analysis of single modified peptides is more difficult compared to protein quantification, where quantitative information is usually derived from at least two peptides.

IMAC- and metal oxide-based methods equally enrich phosphopeptides with phosphoserine, phosphothreonine, and phosphotyrosine residues. If tyrosine kinases and the relatively rare tyrosine phosphorylation sites are of special interest, antiphosphotyrosine antibodies are frequently employed to enrich peptides containing phosphotyrosine for MS analyses. [49]

\subsubsection{Protein Acetylation, Methylation, Ubiquitination and ADP-ribosylation}

Phosphoproteomics and glycoproteomics are important subdisciplines of proteomics, but also acetylation of lysine residues, methylation of lysine and arginine, and ubiquitination of lysine represent widely studied protein modifications. A comprehensive review of large-scale analysis of these prominent PTMs by mass spectrometry was provided by J. Olsen and M. Mann. ${ }^{50]}$ Modification-specific antibodies against acetylated lysine ${ }^{29]}$ and mono- and dimethylated arginine and lysine residues $^{[30]}$ are available and have been successfully utilized in proteome-wide PTM studies.

The attachment of ubiquitin or of ubiquitin-related proteins is another biologically significant protein modification, which was difficult to study for a long time. Protein ubiquitination and polyubiquitination events are not only involved in protein degradation, but also in various other biological processes including gene expression and DNA repair. In most cases, linkages are formed between lysine residues and the $\mathrm{C}$-terminal carboxylic acid of ubiquitin. Trypsin cleavage leaves two C-terminal glycine residues of ubiquitin on the modified lysine residues, and global analysis of lysine ubiquitination was made possible by antibodies against these diglycyl-lysine residues. ${ }^{[31]}$ Thousands of peptides containing ubiquitination sites can now be enriched from complex samples and analyzed by mass spectrometry.

ADP-ribosylation of proteins is an example of a biologically important protein modification, which is rather understudied by proteomic approaches for several reasons: $(i)$ the modification is relatively large and unstable, particularly in its polymeric form; (ii) poly-ADP-ribosylations need to be reduced to monoADP-ribosylations for analytical purposes; (iii) analysis of the modification requires enrichment of respective peptides, which is accomplished by using ADP-ribose-binding protein domains. ETD or ETD-like fragmentation methods, which leave the attached ADP-ribose largely intact, have been applied, allowing accurate localization and assignment of the modified amino acid.[32] Alternatively, analyses were performed using combined HCD and EThcD data sets. ${ }^{[34]}$ The amino acid acceptor sites are still a matter of debate, although serine ${ }^{[32,33]}$ and arginine ${ }^{[34]}$ are now recognized as major ADP-ribosylation sites.

\subsubsection{Glycoproteomics}

Glycosylation is a ubiquitous and heterogeneous posttranslational modification, regulating the activity of proteins in a large number and variety of biological processes. Glycoproteomics, which includes localization and structural analysis of glycans on proteins, is a major subdiscipline of proteomics. The two main types of glycosylation on eukaryotic proteins are $\mathrm{N}$-glycans linked to asparagine at consensus sites and O-glycans occurring mainly on serine and threonine residues. Proteome-wide studies and automated identification of intact glycopeptides comparable to those for other PTMs has been a longstanding goal in glycoproteomics. Proteins are first cleaved with proteases into peptides and glycopeptides, then the samples are either enriched for glycopeptides, for example by lectin affinity chromatography or by hydrophilic interaction chromatography, or directly subjected to LC-MS analysis. In principle, this approach allows identification 
of glycoproteins and of their glycosylations at specific sites, but these proteome-wide studies are still challenging because of the structural diversity of glycans. Several groups recently published improved software tools for this purpose. ${ }^{[51-53]}$

Glycomic studies involve MS-analysis of glycan structures released from proteins. N-glycans are released mainly by enzymatic methods, and O-glycans by chemical or enzymatic methods. Chemical derivatization of glycans, most commonly permethylation, is usually performed, to increase sensitivity and facilitate structural analysis. Glycans may be analyzed by MALDI mass spectrometry or by electrospray mass spectrometry coupled with liquid chromatography. In addition to analyses at the levels of glycans and glycopeptides, direct analysis of intact glycoproteins by high-resolution MS methods was proposed by the group of A. Heck. ${ }^{[35]}$ A very detailed and comprehensive review of mass spectrometric glycomic and glycoproteomic analyses was recently published by L. Ruhaak et al. ${ }^{[36]}$ A schematic of the glycan and glycoproteomics workflows is depicted in Fig. 2.

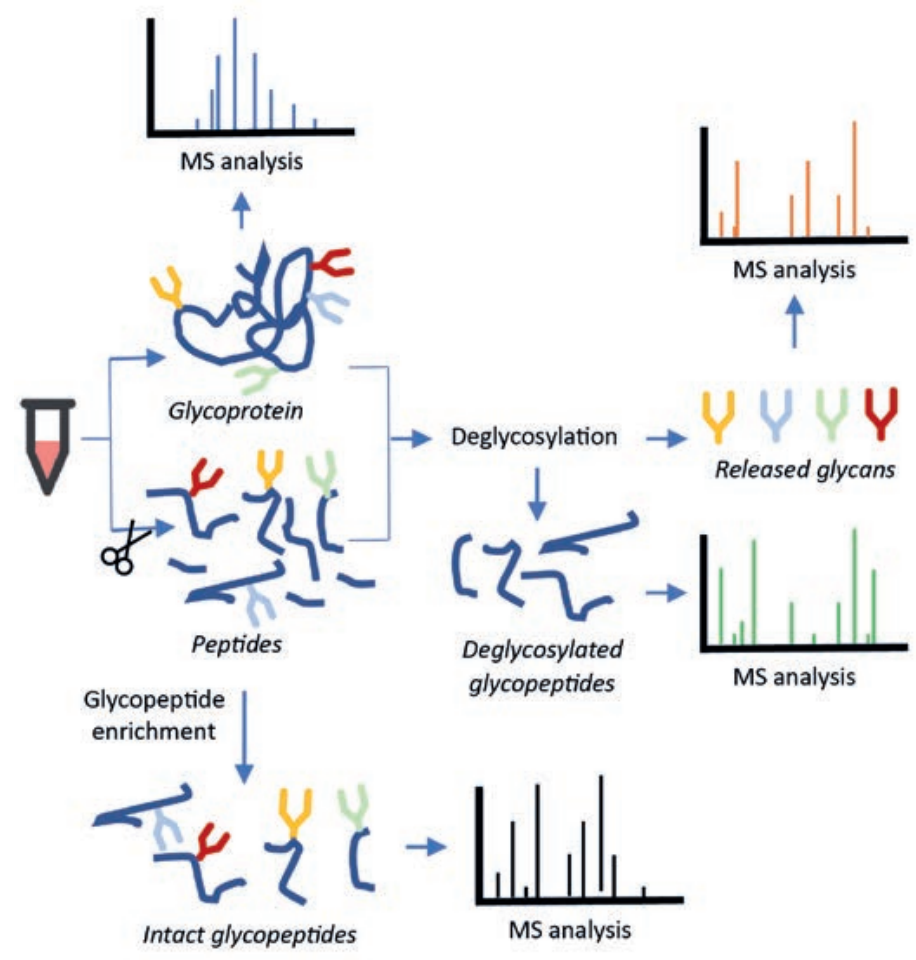

Fig. 2. Schematic of the MS-based workflows for glycan and glycoproteomics analysis.

\subsubsection{Disulfide Bonds}

Disulfide bonds formed between two cysteine residues occur in a significant number of proteins. They have structural and functional roles, and they ensure proper protein folding, hence it is particularly important to confirm correct formation of disulfide bonds in protein therapeutics such as antibodies. Although the assignment of disulfide bonds has been performed by MS for several decades, it is still not a trivial task.

A typical workflow for the analysis of disulfide bonds requires complete alkylation of free cysteines. Cleavage of native disulfide bonds and formation of new bonds may occur during proteolysis, particularly in the presence of free cysteines. Subsequently, proteolytic digestion of the protein using suitable enzymes is performed, avoiding high temperatures and alkaline $\mathrm{pH}$ values to minimize disulfide bond artifacts. The disulfide-linked peptides are then analyzed by mass spectrometry, with the aim to assign the disulfide bridges in the protein. CID and HCD fragmentation mainly result in peptide backbone fragmentation and leave the disulfide bonds intact, while ETD and related fragmentation methods primarily cleave the disulfide bonds. Experimental strategies, LC-MS techniques, and algorithms for disulfide bond assignment have been extensively reviewed by Labkub et al. ${ }^{[37]}$

\subsubsection{Protein N-Termini}

Identification of the N-termini of mature proteins and of $\mathrm{N}$-terminal modifications is an important aspect of protein characterization. $\mathrm{N}$-terminal maturation of proteins includes partial cleavage of the initial methionine, acetylation or the addition of other modifications, and removal of signal peptides. A strategy for the proteome-wide determination of real protein $\mathrm{N}$-termini and of cleavage products of proteases has been developed by $\mathrm{C}$. Overall and others. ${ }^{[38]}$ Their approach, named terminal amine isotopic labeling of substrates (TAILS), includes the following steps: (i) isotopic labeling of untreated proteomes and of proteomes cleaved with an endogenous protease, for example by using iTRAQ or TMT reagents; (ii) mixing of these samples and digestion with trypsin; (iii) capture of internal tryptic peptides with an amine-reactive polymer. In this way, a negative selection of peptides containing blocked N-termini (the peptides containing the N-termini of the original proteins) is achieved. Following this approach, the $\mathrm{N}$-terminal peptides of the mature proteins and those generated by the protease of interest are isolated and can be subjected to MS analysis.

\subsection{Structural Proteomics}

MS-based proteomics is not limited to the generation of protein inventories of biological samples. A number of methods that yield proteome-wide information on structures of proteins and protein complexes have been developed. Limited proteolysis coupled to MS, cross-linking MS, and mass spectrometric detection of noncovalent protein-ligand or protein-protein complexes belong to the most widespread structural proteomics approaches.

\subsubsection{Limited Proteolysis}

Limited proteolysis followed by mass spectrometry analysis (LiP-MS) is a proteomics approach that enables the identification of structural changes of proteins on a proteome-wide scale and directly in biological extracts. ${ }^{[39]}$ Applications of LiP-MS include identification of structural changes of proteins under different conditions, profiling of protein stability, and even identification of protein targets of small molecules. In the LiPMS workflow, proteins are initially extracted from biological samples under native conditions. Protein extracts are subjected to different conditions, and subsequently partially cleaved with an unspecific protease. These partially cleaved samples and control samples are denatured, completely cleaved at lysine and arginine residues, and analyzed and quantified by mass spectrometry. The general idea behind the analysis of LiP-MS data is that protein regions containing LiP cleavage sites are identified by an abundance decrease of the associated fully tryptic peptides or by the appearance or increased intensity of peaks corresponding to peptides with nontryptic termini (half-tryptic peptides) in the native samples compared with that in the control samples.

\subsubsection{Cross-linking Proteomics}

Another rapidly advancing method in structural proteomics is cross-linking mass spectrometry, ${ }^{[40,41]}$ which determinesinteracting proteins by covalent crosslinking of residues in close proximity and MS identification of cross-linked peptides. It provides distance constraints to study the structures of proteins, protein-protein interactions, and even large multiprotein complexes. A typical application of cross-linking MS are studies of the topology of purified protein complexes of known composition. Furthermore, 
the method may provide complementary information for APMS-based interactome studies, particularly if protein interactions are not well-preserved during cell lysis and purification. A large variety of cross-linkers are available, but all of them consist of reactive end groups and spacers of different lengths. The crosslinking MS workflow involves the reaction of cross-linkers with proteins, proteolytic digestion, optional enrichment of crosslinked peptides, and MS analysis. The detection of cross-linked peptides in complex peptide mixtures is still a main difficulty of these experiments.

\subsubsection{Studies of Noncovalent Protein Complexes by Native Mass Spectrometry}

Electrospray ionization is a particularly gentle ionization technique, and it allows the detection of noncovalent proteinprotein and protein-ligand complexes if their native structures are retained in solution and at least partially in the gas phase. In principle, the stoichiometry of protein complexes can be obtained using mass measurements of intact complexes. ${ }^{[42,43]}$ In this respect, studies of non-covalent protein interactions by native MS are complementary to other methods for protein complex analyses. However, there is no general agreement to what extent protein structures in solution can be preserved in the gas phase. Applications of native mass spectrometry include studies of protein heterogeneity, of protein assemblies, and of noncovalent protein interactions with metal ions, lipids, carbohydrates, and drug molecules. Even binding affinities were assessed from these analyses. The field has strongly benefited from technical advances in mass spectrometry, regarding sensitivity, resolution and mass accuracy, and in particular upper limits of the mass range.

\section{Proteome Bioinformatics}

Thanks to the rapid development of MS-based bioanalytical workflows, thousands of proteins can be concurrently measured in a single sample. Each experiment generates a large amount of data that needs to be processed. The results need to be interpreted in the context of the research question (e.g. identification of the proteins involved in the disease development or regulation of a specific cellular mechanism). A plethora of software and bioinformatics methods focusing on different aspects of the MSdata analysis process have been developed over the last years and recently reviewed by Chen et al. ${ }^{[54]}$

The first step of a standard proteomics bioinformatics analysis consists of peptide and protein identification. Search engine software (e.g. Mascot[55] or SEQUEST[6]) matches MS/MS fragmentation spectra, typically acquired using DDA methods, against organism-specific protein sequence databases available in public repositories such as Uniprot, NCBI or Ensembl. Given that most proteomics datasets are very large and lead to the identification of hundreds or thousands of peptides and proteins, the identification results may include false positives. For this reason, a statistical validation is required and a False Discovery Rate (FDR) estimation is always performed to correct for multiple testing of hypotheses. ${ }^{[56]}$

Protein quantification can be performed as an additional step after protein identification. Commonly used software for quantification includes MaxQuant, ${ }^{[57]}$ Spectronaut (Biognosys), Proteome Discoverer (Thermo Scientific) or Skyline. ${ }^{[58]}$ Once a list of proteins with their corresponding quantitative information has been generated, data are usually normalized to remove any non-biological variation; this is a critical process where the right normalization method should be selected depending on the setup and the design of the experiment. After this step, the statistical analysis is performed to detect significant protein abundance changes in conditions of interest by applying statistical tests such as the t-test, ${ }^{[59]}$ ANOVA, ${ }^{[60]}$ or LIMMA. ${ }^{[61]}$ Typically, at this point of the proteomics data analysis, data are subjected to enrichment analysis with the goal to identify proteins that are overrepresented in specific sets of proteins or genes (e.g. a biological process such as signaling). During these steps, it is possible to link the protein lists with the biological events occurring in the studied organism.

\section{Proteomics Infrastructure and Expertise}

The selection of MS-based proteomics applications described above offers solutions for research questions ranging from protein quantification and characterization to the study of protein structures and functions. Each of these applications consists of one or multiple analytical methods and workflows, which allows for a customization of the analytical strategy to the specific biological research question, but poses a significant challenge to identify and implement the optimal approach. Proteomics experts need to have extensive knowledge and experience with many of these workflows, as well as access to appropriate highend MS instrumentation, to successfully design and perform experiments. As proteomics is a highly data-rich analytical area, experts are also required to profoundly understand the structure of the resulting MS data and to be able to apply advanced bioinformatics and statistical methods. Applying MS-based proteomics in biological research is an inherently interdisciplinary activity and using proteomics, even more so the setting up of a proteomics laboratory, is a highly complex task. In the following, we discuss selected practical and strategic aspects for the use or even establishment of proteomics in a research entity. We also illustrate the advantages of collaborating with academic core facilities and service providers for researchers without specialized know-how in the area.

\subsection{Using Proteomics in Research Entities or in Collaboration with Specialized Support Providers}

On the infrastructure side, mass spectrometers are expensive instruments with significant running costs and infrastructure requirements (e.g. heating, noise, space). They need regular maintenance procedures performed either by the members of the research group or by qualified personnel from the instrument vendors. Service and support contracts are expensive and only affordable when instruments are fully utilized. Finally, instruments at the cutting edge are outdated in a few years, with consequences for amortization and re-investment timelines. On the personnel side, trained specialists are required to operate and maintain the MS systems and to use them optimized to the research question at hand, including data processing and data analysis workflows. To establish the respective knowledge easily takes years and requires multiple experts to cover the broad field of proteomics. It is therefore problematic if research groups that are not primarily interested in proteomics research, establish the respective expertise with temporary staff at the $\mathrm{PhD}$ student or post-doc level, who leave the entity after a few years.

As a consequence, most research groups in the life sciences rely on third parties for the mass spectrometry or even larger proteomics parts of their projects. Researchers from industry often use commercial proteomics services, for which a small set of specialized providers exist. Academic researchers may search for collaboration with specialized research groups having their own MS instruments and application expertise, which are often developing new workflows or even instrumentations. Academic core facilities are a support structure in between service providers and collaborations. By combining the research needs of whole universities or large research institutions, core facilities focus on optimal use of equipment and capacity, and on the provision of a large portfolio of standard and customized applications, ranging from proteomics, to protein analysis, metabolomics, ${ }^{62]}$ lipidomics ${ }^{[63]}$ and even glycomics. ${ }^{[35,36]}$ The specialized service and support organizations, be it commercial or academic, take care of long-term know-how establishment, expansion and securement, 
as well as the capital expenses for equipment, software, and data processing hardware and infrastructures.

\subsection{Training of Proteomics Scientists}

To cover the know-how gap between experts in the field and researchers in need of proteomics data but without a MS or analytical focus, an increasing number of academic institutions offer courses covering bioanalytical applications of mass spectrometry, including sample preparation, data acquisition and data analysis. These courses are mostly concentrated within a few weeks and lay an important basis for the education of future expert users of proteomics. Beyond this level, practical and extensive hands-on experience can be gained at academic core facilities through their training of life scientists running longterm MS projects. In these cases, steps of the workflows can be extensively trained and practiced, leading to expert users that can better understand the structure of the data and the potential of the methods, including their pitfalls. These scientists are then in the best-possible position to make optimal use of proteomics technologies and methods, and to extract knowledge from the resulting data, applying their specialized expertise on the biological system under investigation.

\section{Conclusions and Outlook}

Advances in mass spectrometry-based protein analysis and proteomics methods are contributing to a better understanding of biological processes in most life-science fields. Important technological and analytical improvements have been achieved over the last years, increasing the sensitivity of the methods and expanding the horizon of MS-based proteomics applications. The field of single-cell and low-input proteomics is now becoming accessible, ${ }^{[64]}$ allowing to apply mass spectrometry to projects studying heterogeneous cell populations or other biological samples where large protein amounts are not always available (e.g. the systematic profiling of organelles and protein complexes, or the study of low abundant protein modifications). Finally, the development of bioinformatics solutions is enabling the integration of proteomics data with the results from other omics fields such as metabolomics, lipidomics and transcriptomics. Integrating these types of data enables scientists to answer both clinical and fundamental research questions of higher complexity, with increased scientific confidence and accuracy. The combined improvements and new possibilities led to a significant growth of the proteomics field and an increase in the number of academic and industrial research groups requiring access to proteomics instrumentation and knowledge. As a consequence, the technical and scientific progress has been paralleled by the organizational development of proteomics core facilities. They play a fundamental role by providing infrastructure, expertise, services and trainings and continue to contribute to the present and future increased impact of proteomics on basic and applied research.

\section{Acknowledgements}

This work was supported by ETH Zurich and University of Zurich. The authors declare no conflict of interest.

Received: November 1, 2021

[1] K. Biemann, G. Gapp, J. Seibl, J. Am. Chem. Soc. 1959, 81, 2274, https://doi.org/10.1021/ja01518a069.

[2] K. Biemann, J. Proteomics 2014, 107, 62, https://doi.org/10.1016/j.jprot.2014.01.008.

[3] J. B. Fenn, M. Mann, C. K. Meng, S. F. Wong, C. M. Whitehouse, Science 1989, 246, 64, https://doi.org/10.1126/science.2675315.

[4] M. Karas, F. Hillenkamp, Anal. Chem. 1988, 60, 2299, https://doi.org/10.1021/ac00171a028.

[5] R. A. Henderson, H. Michel, K. Sakaguchi, J. Shabanowitz, E. Appella, D. F. Hunt, V. H. Engelhard, Science 1992, 255, 1264, https://doi.org/10.1126/science.1546329.
[6] J. K. Eng, A. L. McCormack, J. R. Yates, J. Am. Soc. Mass Spectrom. 1994, 5, 976, https://doi.org/10.1016/1044-0305(94)80016-2.

[7] J. C. Schwartz, M. W. Senko, J. E. Syka, J. Am. Soc. Mass Spectrom. 2002, 13, 659, https://doi.org/10.1016/S1044-0305(02)00384-7.

[8] H. R. Morris, T. Paxton, A. Dell, J. Langhorne, M. Berg, R. S. Bordoli, J. Hoyes, R. H. Bateman, Rapid Commun. Mass Spectrom. 1996, 10, 889, https://doi.org/10.1002/(SICI) 1097-0231(19960610)10:8<889::AIDRCM615>3.0.CO;2-F.

[9] A. Makarov, Anal. Chem. 2000, 72, 1156, https://doi.org/10.1021/ac991131p.

[10] A. Michalski, E. Damoc, J. P. Hauschild, O. Lange, A. Wieghaus, A. Makarov, N. Nagaraj, J. Cox, M. Mann, S. Horning, Mol. Cell Proteomics 2011, 10, M111 011015, https://doi.org/10.1074/mcp.M111.011015.

[11] M. W. Senko, P. M. Remes, J. D. Canterbury, R. Mathur, Q. Song, S. M. Eliuk, C. Mullen, L. Earley, M. Hardman, J. D. Blethrow, H. Bui, A. Specht, O. Lange, E. Denisov, A. Makarov, S. Horning, V. Zabrouskov, Anal. Chem. 2013, 85, 11710, https://doi.org/10.1021/ac403115c.

[12] F. Meier, A. D. Brunner, S. Koch, H. Koch, M. Lubeck, M. Krause, N. Goedecke, J. Decker, T. Kosinski, M. A. Park, N. Bache, O. Hoerning, J. Cox, O. Rather, M. Mann, Mol. Cell Proteomics 2018, 17, 2534, https://doi.org/10.1074/mcp.TIR118.000900.

[13] S. Pfammatter, E. Bonneil, F. P. McManus, S. Prasad, D. J. Bailey, M. Belford, J. J. Dunyach, P. Thibault, Mol. Cell Proteomics 2018, 17, 2051, https://doi.org/10.1074/mcp.TIR118.000862.

[14] J. E. Syka, J. J. Coon, M. J. Schroeder, J. Shabanowitz, D. F. Hunt, Proc. Natl. Acad. Sci. USA 2004, 101, 9528, https://doi.org/10.1073/pnas.0402700101.

[15] J. V. Olsen, B. Macek, O. Lange, A. Makarov, S. Horning, M. Mann, Nat. Methods 2007, 4, 709, https://doi.org/10.1038/nmeth1060.

[16] C. K. Frese, A. F. Altelaar, H. van den Toorn, D. Nolting, J. GriepRaming, A. J. Heck, S. Mohammed, Anal. Chem. 2012, 84, 9668, https://doi.org/10.1021/ac3025366.

[17] D. A. Wolters, M. P. Washburn, J. R. Yates, 3rd, Anal. Chem. 2001, 73, 5683, https://doi.org/10.1021/ac010617e.

[18] S. E. Ong, M. Mann, Methods Mol. Biol. 2007, 359, 37, https://doi.org/10.1007/978-1-59745-255-7_3.

[19] P. L. Ross, Y. N. Huang, J. N. Marchese, B. Williamson, K. Parker, S. Hattan, N. Khainovski, S. Pillai, S. Dey, S. Daniels, S. Purkayastha, P. Juhasz, S. Martin, M. Bartlet-Jones, F. He, A. Jacobson, D. J. Pappin, Mol. Cell Proteomics 2004, 3, 1154, https://doi.org/10.1074/mcp.M400129-MCP200.

[20] A. Thompson, J. Schafer, K. Kuhn, S. Kienle, J. Schwarz, G. Schmidt, T. Neumann, R. Johnstone, A. K. Mohammed, C. Hamon, Anal. Chem. 2003 75,1895 , https://doi.org/10.1021/ac0262560.

[21] J. Cox, M. Y. Hein, C. A. Luber, I. Paron, N. Nagaraj, M. Mann, Mol. Cell Proteomics 2014, 13, 2513, https://doi.org/10.1074/mcp.M113.031591.

[22] P. Picotti, R. Aebersold, Nat. Methods 2012, 9, 555 , https://doi.org/10.1038/nmeth.2015.

[23] A. C. Peterson, J. D. Russell, D. J. Bailey, M. S. Westphall, J. J. Coon, Mol. Cell Proteomics 2012, 11, 1475, https://doi.org/10.1074/mcp.O112.020131.

[24] L. C. Gillet, P. Navarro, S. Tate, H. Rost, N. Selevsek, L. Reiter, R. Bonner, R. Aebersold, Mol. Cell Proteomics 2012, 11, O111 016717, https://doi.org/10.1074/mcp.O111.016717.

[25] A. C. Gingras, M. Gstaiger, B. Raught, R. Aebersold, Nat. Rev. Mol. Cell Biol. 2007, 8, 645, https://doi.org/10.1038/nrm2208.

[26] K. J. Roux, D. I. Kim, M. Raida, B. Burke, J. Cell Biol. 2012, 196, 801, https://doi.org/10.1083/jcb.201112098.

[27] S. Han, N. D. Udeshi, T. J. Deerinck, T. Svinkina, M. H. Ellisman, S. A. Carr, A. Y. Ting, Cell Chem. Biol. 2017, 24, 404, https://doi.org/10.1016/j.chembiol.2017.02.002.

[28] N. M. Riley, J. J. Coon, Anal. Chem. 2016, 88, 74, https://doi.org/10.1021/acs.analchem.5b04123.

[29] S. C. Kim, R. Sprung, Y. Chen, Y. Xu, H. Ball, J. Pei, T. Cheng, Y. Kho, H. Xiao, L. Xiao, N. V. Grishin, M. White, X. J. Yang, Y. Zhao, Mol. Cell 2006 , 23, 607, https://doi.org/10.1016/j.molcel.2006.06.026.

[30] A. Guo, H. Gu, J. Zhou, D. Mulhern, Y. Wang, K. A. Lee, V. Yang, M. Aguiar, J. Kornhauser, X. Jia, J. Ren, S. A. Beausoleil, J. C. Silva, V. Vemulapalli, M. T. Bedford, M. J. Comb, Mol. Cell Proteomics 2014, 13, 372, https://doi.org/10.1074/mcp.O113.027870.

[31] G. Xu, J. S. Paige, S. R. Jaffrey, Nat. Biotechnol. 2010, 28, 868 , https://doi.org/10.1038/nbt.1654.

[32] S. C. Larsen, I. A. Hendriks, D. Lyon, L. J. Jensen, M. L. Nielsen, Cell Rep. 2018, 24, 2493, https://doi.org/10.1016/j.celrep.2018.07.083.

[33] O. Leidecker, J. J. Bonfiglio, T. Colby, Q. Zhang, I. Atanassov, R. Zaja, L. Palazzo, A. Stockum, I. Ahel, I. Matic, Nat. Chem. Biol. 2016, 12, 998, https://doi.org/10.1038/nchembio.2180.

[34] M. Leutert, S. Menzel, R. Braren, B. Rissiek, A. K. Hopp, K. Nowak, L. Bisceglie, P. Gehrig, H. Li, A. Zolkiewska, F. Koch-Nolte, M. O. Hottiger, Cell Rep. 2018, 24, 1916, https://doi.org/10.1016/j.celrep.2018.07.048

[35] Y. Yang, V. Franc, A. J. R. Heck, Trends Biotechnol. 2017, 35, 598, https://doi.org/10.1016/j.tibtech.2017.04.010.

[36] L. R. Ruhaak, G. Xu, Q. Li, E. Goonatilleke, C. B. Lebrilla, Chem. Rev. 2018, 118, 7886, https://doi.org/10.1021/acs.chemrev.7b00732. 
[37] J. C. Lakbub, J. T. Shipman, H. Desaire, Anal. Bioanal. Chem. 2018, 410 2467, https://doi.org/10.1007/s00216-017-0772-1.

[38] O. Kleifeld, A. Doucet, U. auf dem Keller, A. Prudova, O. Schilling, R. K. Kainthan, A. E. Starr, L. J. Foster, J. N. Kizhakkedathu, C. M. Overall, Nat. Biotechnol. 2010, 28, 281, https://doi.org/10.1038/nbt.1611.

[39] Y. Feng, G. De Franceschi, A. Kahraman, M. Soste, A. Melnik, P. J. Boersema, P. P. de Laureto, Y. Nikolaev, A. P. Oliveira, P. Picotti, Nat. Biotechnol. 2014, 32, 1036, https://doi.org/10.1038/nbt.2999.

[40] F. J. O'Reilly, J. Rappsilber, Nat. Struct. Mol. Biol. 2018, 25, 1000, https://doi.org/10.1038/s41594-018-0147-0.

[41] A. Leitner, M. Faini, F. Stengel, R. Aebersold, Trends Biochem. Sci. 2016, 41, 20, https://doi.org/10.1016/j.tibs.2015.10.008.

[42] J. A. Loo, Mass Spectrom. Rev. 1997, 16, 1, https://doi.org/10.1002/ (SICI) 1098-2787(1997)16:1<1::AID-MAS1>3.0.CO;2-L.

[43] S. Tamara, M. A. den Boer, A. J. R. Heck, Chem. Rev. 2021, https://doi.org/10.1021/acs.chemrev.1c00212.

[44] A. L. Richards, M. Eckhardt, N. J. Krogan, Mol. Syst. Biol. 2021, 17, e8792, https://doi.org/10.15252/msb.20188792.

[45] P. Samavarchi-Tehrani, R. Samson, A. C. Gingras, Mol. Cell Proteomics 2020, 19, 757, https://doi.org/10.1074/mcp.R120.001941.

[46] C. T. Walsh, S. Garneau-Tsodikova, G. J. Gatto, Jr., Angew. Chem. Int. Ed. 2005, 44, 7342, https://doi.org/10.1002/anie.200501023.

[47] S. B. Ficarro, M. L. McCleland, P. T. Stukenberg, D. J. Burke, M. M. Ross, J. Shabanowitz, D. F. Hunt, F. M. White, Nat. Biotechnol. 2002, 20, 301, https://doi.org/10.1038/nbt0302-301.

[48] S. A. Beausoleil, M. Jedrychowski, D. Schwartz, J. E. Elias, J. Villen, J. Li, M. A. Cohn, L. C. Cantley, S. P. Gygi, Proc. Natl. Acad. Sci. USA 2004, 101, 12130, https://doi.org/10.1073/pnas.0404720101.

[49] J. Rush, A. Moritz, K. A. Lee, A. Guo, V. L. Goss, E. J. Spek, H. Zhang, X. M. Zha, R. D. Polakiewicz, M. J. Comb, Nat. Biotechnol. 2005, 23, 94, https://doi.org/10.1038/nbt1046.

[50] J. V. Olsen, M. Mann, Mol. Cell Proteomics 2013, 12, 3444, https://doi.org/10.1074/mcp.O113.034181.

[51] J. Stadlmann, J. Taubenschmid, D. Wenzel, A. Gattinger, G. Durnberger, F. Dusberger, U. Elling, L. Mach, K. Mechtler, J. M. Penninger, Nature 2017, 549, 538, https://doi.org/10.1038/nature24015.

[52] D. A. Polasky, F. Yu, G. C. Teo, A. I. Nesvizhskii, Nat. Methods 2020, 17, 1125, https://doi.org/10.1038/s41592-020-0967-9.

[53] L. Lu, N. M. Riley, M. R. Shortreed, C. R. Bertozzi, L. M. Smith, Nat. Methods 2020, 17, 1133, https://doi.org/10.1038/s41592-020-00985-5.
[54] C. Chen, J. Hou, J. J. Tanner, J. Cheng, Int. J. Mol. Sci. 2020, 21 , https://doi.org/10.3390/ijms21082873.

[55] D. N. Perkins, D. J. Pappin, D. M. Creasy, J. S. Cottrell, Electrophoresis 1999, 20, 3551 , https://doi.org/10.1002/(SICI)1522-2683(19991201)20:18<3551::AIDELPS3551>3.0.CO;2-2

[56] H. Choi, A. I. Nesvizhskii, J. Proteome Res. 2008, 7, 47, https://doi.org/10.1021/pr700747q.

[57] J. Cox, M. Mann, Nat. Biotechnol. 2008, 26, 1367, https://doi.org/10.1038/nbt.1511.

[58] B. MacLean, D. M. Tomazela, N. Shulman, M. Chambers, G. L. Finney, B. Frewen, R. Kern, D. L. Tabb, D. C. Liebler, M. J. MacCoss, Bioinformatics 2010, 26, 966, https://doi.org/10.1093/bioinformatics/btq054

[59] M. Krzywinski, N. Altman, Nat. Methods 2013, 10, 1041 https://doi.org/10.1038/nmeth.2698.

[60] M. L. McHugh, Biochem. Med. (Zagreb) 2011, 21, 203, https://doi.org/10.11613/bm.2011.029.

[61] K. Kammers, R. N. Cole, C. Tiengwe, I. Ruczinski, EuPA Open Proteom. 2015, 7, 11, https://doi.org/10.1016/j.euprot.2015.02.002.

[62] C. H. Johnson, J. Ivanisevic, G. Siuzdak, Nat. Rev. Mol. Cell Biol. 2016, 17, 451, https://doi.org/10.1038/nrm.2016.25.

[63] T. Zullig, M. Trotzmuller, H. C. Kofeler, Anal. Bioanal. Chem. 2020, 412 , 2191, https://doi.org/10.1007/s00216-019-02241-y.

[64] R. T. Kelly, Mol. Cell Proteomics 2020, 19, 1739 , https://doi.org/10.1074/mcp.R120.002234.

\section{License and Terms}

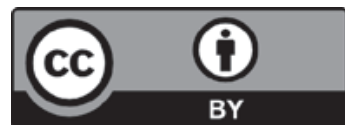

This is an Open Access article under the terms of the Creative Commons Attribution License CC BY 4.0. The material may not be used for commercial purposes

The license is subject to the CHIMIA terms and conditions: (https://chimia.ch/chimia/about)

The definitive version of this article is the electronic one that can be found at https://doi.org/10.2533/chimia.2022.73 